UCRL-ID-125094

\title{
Time Averaging of Instantaneous Quantities in HYDRA
}

\author{
Rose C. McCallen \\ SOEVED \\ DEC $O 91996$ \\ OSTI
}

September 25, 1996

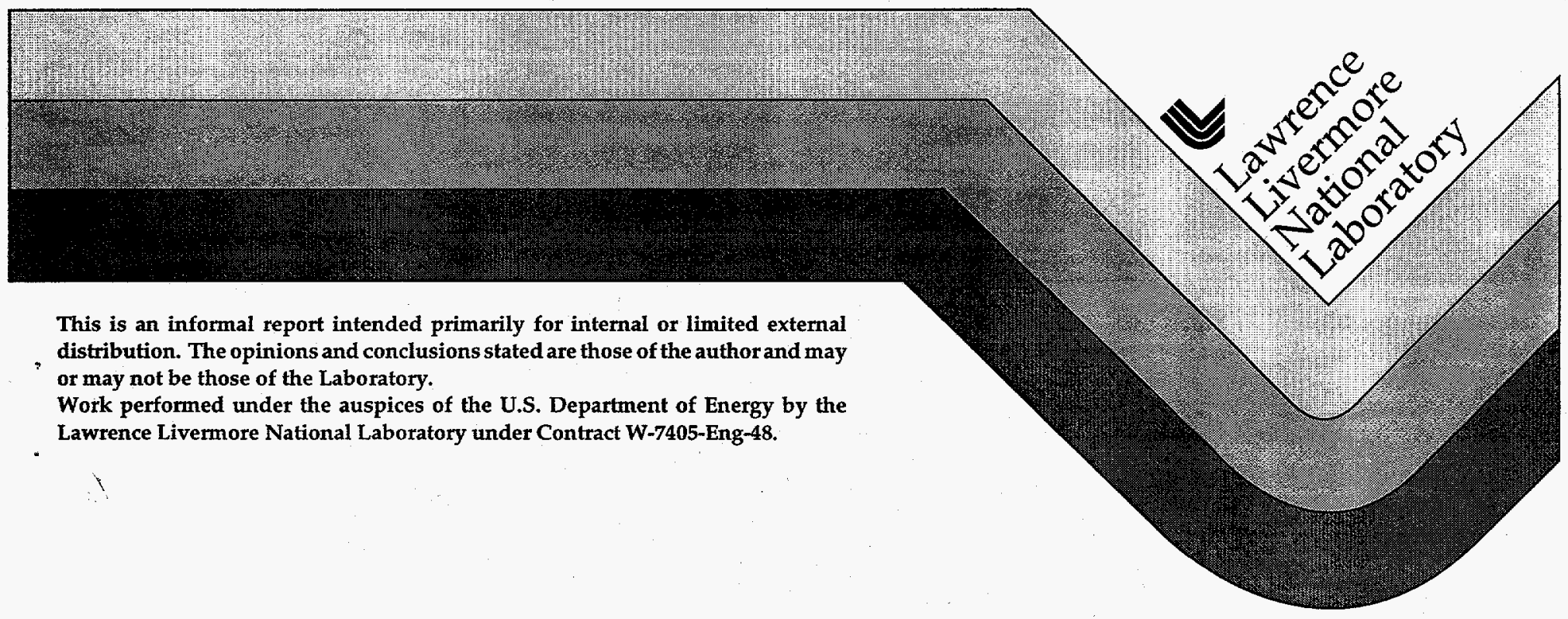




\section{DISCLAIMER}

This document was prepared as an account of work sponsored by an agency of the United States Government. Neither the United States Government nor the University of California nor any of their employees, makes any warranty, express or implied, or assumes any legal liability or responsibility for the accuracy, completeness, or usefulness of any information, apparatus, product, or process disclosed, or represents that its use would not infringe privately owned rights. Reference herein to any specific commercial product, process, or service by trade name, trademark, manufacturer, or otherwise, does not necessarily constitute or imply its endorsement, recommendation, or favoring by the United States Government or the University of California. The views and opinions of authors expressed herein do not necessarily state or reflect those of the United States Government or the University of California, and shall not be used for advertising or product endorsement purposes.

This report has been reproduced directly from the best available copy.

Available to DOE and DOE contractors from the Office of Scientific and Technical Information P.O. Box 62, Oak Ridge, TN 37831

Prices available from (615) 576-8401, FTS 626-8401

Available to the public from the

National Technical Information Service

U.S. Department of Commerce 5285 Port Royal Rd.,

Springfield, VA 22161 


\section{DISCLAIMIER}

Portions of this document may be illegible in electronic image products. Images are produced from the best available original document. 


\title{
Time Averaging of Instantaneous Quantities in HYDRA
}

\author{
Rose C. McCallen
}

\subsection{Background}

For turbulent flow the evaluation of direct numerical simulations (DNS) where all scales are resolved and large-eddy simulations (LES) where only large-scales are resolved is difficult because the results are three-dimensional and transient. To simplify the analysis, the instantaneous flow field can be averaged in time for evaluation and comparison to experimental results.

The incompressible Navier-Stokes flow code HYDRA (Christon 1995) has been modified for calculation of time-average quantities for both DNS and LES. The following describes how time averages of the instantaneous quantities are generated during program execution (i.e., while generating the instantaneous quantities, instead of as a postprocessing operation). The calculations are performed during program execution to avoid storing values at each time step and thus to reduce storage requirements. The method used in calculating the time-average velocities, turbulent intensities, $\left\langle u^{\prime \prime 2}\right\rangle,\left\langle v^{\prime \prime}{ }^{2}\right\rangle$, and $\left\langle w^{\prime \prime 2}\right\rangle$, and turbulent shear, $\left\langle u^{\prime \prime} v^{\prime \prime}\right\rangle$ are outlined. The brackets $>$ used here represent a time average. The described averaging methods were implemented in the HYDRA code for three-dimensional problem solutions. Also presented is a method for taking the time averages for a number of consecutive intervals and calculating the time average for the sum of the intervals. This method could be used for code restarts or further postprocessing of the time averages from consecutive intervals. This method was not used in the HYDRA implementation, but is included here for completeness. In HYDRA, the running sums needed for time averaging are simply written to the restart dump.

\subsection{Time-Average Definitions}

The instantaneous velocity field ${ }^{1}$ can be decomposed into a mean velocity $\langle u\rangle$ (time, space, or ensemble averaged) and a fluctuating velocity, $u^{\prime \prime}$, such that the instantaneous velocity $u$ is defined as

$$
u=\langle u\rangle+u^{\prime \prime}
$$

The averaging operation $<$ is defined such that $\left\langle u^{\prime \prime}\right\rangle=0$. If the turbulent flow field is stationary (i.e., statistically steady), averaging with respect to time can be used (Hinze 1975 or Tennekes and Lumley 1972). By definition,

1. We use the notation $u, v$, and $w$ for the three velocity components in the $x, y$, and $z$ directions. 


$$
<u(\underline{x})>=\lim _{T \rightarrow \infty} \frac{1}{2 T} \int_{-T}^{+T} u(\underline{x}, t) d t
$$

For a finite value of the averaging interval $T$, we define the averaging operator by

$$
<u(\underline{x})>=\frac{1}{T} \int_{t-\frac{T}{2}}^{t+\frac{T}{2}} u\left(\underline{x}, t^{\prime}\right) d t^{\prime}
$$

with the condition that $T_{1}<<T<T_{2}$ (Hinze 1975) where the lower value $T_{1}$ is the time scale for the turbulence and the upper value $T_{2}$ is the period for slow variations in the flow field that we do not regard as part of the turbulence. If the averaging interval $T$ meets the above condition, the average value should be independent of when you start the average. Thus, $\partial\langle u\rangle / \partial t$ is zero (or negligibly small) in the interval $T$ and even zero over several $T$ intervals (since $T<<T_{2}$ ).

In the numerical simulation, the appropriate choice of the averaging interval $T$, such that $T_{1}<<T<T_{2}$, is not known until after the instantaneous velocities have been calculated over a relatively long time interval (i.e., for several $T_{2}$ intervals). However, to avoid storing the velocity field at every time step for postprocessing of the average quantities, we calculate the averages during program execution (i.e., while we calculate the instantaneous velocity). The following describes the method used to calculate time averages during program execution for a chosen $a$ priori interval $T$. If this chosen interval does not produce a time invariant average value or one that varies only slightly over several $T$ intervals, the values for interval $T$ can be postprocessed. As described in the following sections, the averages for $k$ intervals of time duration $T$ can be used to generate vaues for an interval $k T$, where $k$ is a positive integer.

If the chosen $T$ is too small and the time average varies more than desired between adjacent intervals, the calculated time average is dependent on where the interval is started. The time-average velocity is then

$$
\langle u(\underline{x}, t)\rangle=\frac{1}{T} \int_{t-\frac{T}{2}}^{t+\frac{T}{2}} u\left(\underline{x}, t^{\prime}\right) d t^{\prime}
$$

where the value is defined at time $t$ which is the midpoint for the interval $T$. Although the calculated time-average velocities, turbulent intensities, and shear are not the desired result, they can be postprocessed to obtain the time average for a longer time interval.

\subsection{Time-Averaged Quantities}

The methods used in calculating the time-averaged quantities are outlined in the following sections.

Time Averaging of Instantaneous Quantities in HYDRA

September 25, 1996 


\subsection{Time-Averaged Velocities}

The time-averaged velocity $<u>$ for an interval $T$ is defined as

$$
<u(\underline{x}, t)\rangle=\frac{1}{T} \int_{t-\frac{T}{2}}^{t+\frac{T}{2}} u\left(\underline{x}, t^{\prime}\right) d t^{\prime}
$$

and is the value at the interval midpoint $t$. The above integration can be approximated with the discrete summation

$$
\begin{aligned}
& \int_{t-\frac{T}{2}}^{t+\frac{T}{2}} u\left(\underline{x}, t^{\prime}\right) d t^{\prime}=\int_{t-\frac{T}{2}}^{t-\frac{T}{2}+\Delta t_{1}} u\left(\underline{x}, t^{\prime}\right) d t^{\prime}+\int_{t-\frac{T}{2}+\Delta t_{1}}^{t-\frac{T}{2}+\Delta t_{1}+\Delta t_{2}} u\left(\underline{x}, t^{\prime}\right) d t^{\prime}+\ldots+\int_{t+\frac{T}{2}-\Delta t_{n}}^{t+\frac{T}{2}} u\left(\underline{x}, t^{\prime}\right) d t^{\prime} \\
& \\
& \cong u\left(\underline{x}, t-\frac{T}{2}+\frac{\Delta t_{1}}{2}\right) \Delta t_{1}+u\left(\underline{x}, t-\frac{T}{2}+\Delta t_{1}+\frac{\Delta t_{2}}{2}\right) \Delta t_{2}+\ldots+u\left(\underline{x}, t-\frac{T}{2}+\frac{\Delta t_{n}}{2}\right) \Delta t_{n}
\end{aligned}
$$

The above discrete summation (i.e., midpoint rule) is used for the time integration in the numerical calculation, and the averaging is performed using a constant time step to give

$$
<u(\underline{x}, t)>\equiv \frac{\sum_{i=1}^{n} u\left(\underline{x}, t_{i}\right) \cdot(\Delta t)_{i}}{\sum_{i=1}^{n}(\Delta t)_{i}}=\frac{\sum_{i=1}^{n} u_{i}}{n}
$$

where $u_{i}$ is the value of $u$ at time step $i$ and at time $t_{i}, n$ is the number of time steps, and $(\Delta t)_{i}$ is the integration step size which is constant in the existing code (i.e., $\sum(\Delta t)_{i}=n \Delta t=T$ ). This is the time-averaged velocity at the midpoint of the interval and is calculated for every node. Because the summed values are at the midpoint of the interval according to the midpoint rule, the start of the averaging interval is $t-(T / 2)=t_{1}-\left(\Delta t_{1} / 2\right)$ and the end of the averaging interval is $t+(T / 2)=t_{n}+\left(\Delta t_{n} / 2\right)$.

If a longer time interval for averaging is desired, say $k T$ where $k$ is an integer, the values for interval $k T$ can be postprocessed using the following calculation. For time interval $k T$, the timeaverage velocity is 


$$
\begin{aligned}
\langle u(\underline{x}, t)\rangle=\frac{1}{k T} \int_{t-\frac{k T}{2}}^{t+\frac{k T}{2}} u\left(\underline{x}, t^{\prime}\right) d t^{\prime} \cong \frac{\sum_{i=1}^{k n} u_{i}}{k n}=\frac{\sum_{i=1}^{n} u_{i}+\sum_{i=n+1}^{2 n} u_{i}+\ldots+\sum_{i=(k-1) n+1}^{k n} u_{i}}{k n} \\
=\frac{1}{k}\left(\left\langle u>_{1}+\langle u\rangle_{2}+\ldots+\langle u\rangle_{k}\right)=\frac{\left.\sum_{i=1}^{k n}<u\right\rangle_{i}}{k}\right.
\end{aligned}
$$

where $\langle u\rangle_{i}$ is the time-averaged velocity for the time interval $t+(i-1) T-\frac{T}{2}$ to $t+(i-1) T+\frac{T}{2}$

\subsection{Turbulent Intensities}

The $\mathrm{x}$-component of the velocity fluctuation $u^{\prime \prime}$ is defined as

$$
u^{\prime \prime}=u-<u>
$$

and the $\mathrm{x}$-component of the turbulent intensity is

$$
<u^{\prime \prime 2}>=\frac{1}{T} \int_{t-\frac{T}{2}}^{t+\frac{T}{2}} u^{\prime \prime 2} d t^{\prime}=\frac{1}{T} \int_{t-\frac{T}{2}}^{t+\frac{T}{2}}(u-<u>)^{2} d t^{\prime}
$$

If we chose $T$ such that $T_{1}<<T<T_{2}$, we have

$$
<\langle u\rangle\rangle=\langle u\rangle
$$

and for the turbulence intensity

$$
\begin{gathered}
\left.<u^{\prime \prime 2}\right\rangle=\left\langle\left(u-\langle u>)^{2}\right\rangle=\left\langle u^{2}-2<u>u+<u\right\rangle^{2}\right\rangle \\
\left.=\left\langle u^{2}>-2<u\right\rangle\langle u\rangle+<u\right\rangle^{2}=\left\langle u^{2}>-<u\right\rangle^{2}
\end{gathered}
$$

In the numerical calculation, we use the discrete summation for the time integration of $u^{2}$ and thus the expression for calculating $\left\langle u^{\prime \prime 2}\right\rangle$ is

$$
<u^{\prime \prime 2}>\cong \frac{\sum_{i=1}^{n} u_{i}^{2}}{n}-<u>^{2} .
$$


If a longer time interval for averaging is desired, say $k T$ where $k$ is an integer, the intermediate values for interval $k T$ can be postprocessed using the following calculation. For time interval $k T$, the turbulent intensity is

$$
<u^{\prime \prime 2}>=\frac{1}{k T} \int_{t-\frac{k T}{2}}^{t+\frac{k T}{2}} u^{\prime \prime 2} d t^{\prime}
$$

Thus, we have

$$
<u^{\prime \prime 2}>\cong \frac{\sum_{i=1}^{k n} u_{i}^{2}}{k n}-<u>2
$$

where

$$
\frac{\sum_{i=1}^{k n} u_{i}^{2}}{k n}=\frac{1}{k}\left(\frac{\sum_{i=1}^{n} u_{i}^{2}}{n}+\frac{\sum_{i=n+1}^{2 n} u_{i}^{2}}{n}+\ldots+\frac{\sum_{i=(k-1) n+1}^{k n} u_{i}^{2}}{n}\right)
$$

and

$$
\left.<u>^{2} \cong \frac{1}{k^{2}}\left(<u>_{1}+<u>_{2}+\ldots+<u\right\rangle_{k}\right)^{2}
$$

There is no need to save for postprocessing the sum of the squared velocity because we can calculate it from the output average velocity and turbulent intensity as follows:

$$
\begin{aligned}
& \sum_{i=1}^{n} u_{i}^{2} \\
& \frac{i=1}{n}=\left\langle u^{\prime \prime 2}\right\rangle_{1}+\langle u\rangle_{1}^{2} \text {, } \\
& \sum^{n} u_{i}^{2} \\
& \frac{i=n+1}{n}=\left\langle u^{\prime \prime 2}\right\rangle_{2}+\langle u\rangle_{2}^{2} \text {, etc. }
\end{aligned}
$$

Thus, the turbulent intensity for the interval $t-\frac{k T}{2}$ to $t+\frac{k T}{2}$ can be postprocessed from the $k$ intermediate intervals using the following expression

$$
<u^{\prime \prime 2}>\cong \frac{1}{k}<u^{\prime \prime 2}>1+<u>_{1}^{2}+<u^{\prime \prime 2}>2+<u>_{2}^{2}
$$




$$
+\ldots+\left\langle u^{\prime 2}>_{k}+\left\langle u>_{k}^{2}\right)-\frac{1}{k^{2}}\left(\left\langle u>_{1}+\left\langle u>_{2}+\ldots+<u\right\rangle_{k}\right)^{2}\right.\right.
$$

or in summation notation,

$$
\left.\left.<u^{\prime \prime 2}>\cong \frac{1}{k}\left(\sum_{i=1}^{k}<u^{\prime 2}\right\rangle_{i}+\sum_{i=1}^{k}<u>_{i}^{2}-\frac{1}{k}\left(\sum_{i=1}^{k}<u\right\rangle_{i}\right)^{2}\right)
$$

\subsection{Turbulent Shear}

The turbulent shear is defined as

$$
<u^{\prime \prime} v^{\prime \prime}>=\frac{1}{T} \int_{t-\frac{T}{2}}^{t+\frac{T}{2}} u^{\prime \prime} v^{\prime \prime} d t^{\prime}=\frac{1}{T} \int_{t-\frac{T}{2}}^{t+\frac{T}{2}}(u-<u>)(v-<v>) d t^{\prime}
$$

and in the numerical calculation, we have

$$
<u^{\prime \prime} v^{\prime \prime}>\cong \frac{\sum_{i=1}^{n} u_{i} v_{i}}{n}-<u><v>
$$

If a longer time interval for averaging is desired, say $k T$ where $k$ is an integer, the values for interval $k T$ can be postprocessed using the following calculation. For the time interval $k T$

$$
<u^{\prime \prime} v^{\prime \prime}>=\frac{1}{k T} \int_{t-\frac{k T}{2}}^{t+\frac{k T}{2}} u^{\prime \prime} v^{\prime \prime} d t^{\prime} \cong \frac{\sum_{i=1}^{k n} u_{i} v_{i}}{k n}-<u><v>
$$

where

$$
\sum_{i=1}^{k n} u_{i} v_{i}=\frac{1}{k}\left(\frac{\sum_{i=1}^{n} u_{i} v_{i} \sum_{i=n+1}^{2 n} u_{i} v_{i}}{n}+\ldots+\frac{\sum_{i=(k-1) n+1}^{k n} u_{i} v_{i}}{n}\right)
$$

and

$$
\langle u\rangle\langle v\rangle \cong \frac{1}{k^{2}}\left(\langle u\rangle_{1}+\langle u\rangle_{2}+\ldots+\langle u\rangle_{k}\right)\left(\left\langle v>_{1}+\langle v\rangle_{2}+\ldots+\langle v\rangle_{k}\right)\right.
$$


There is no need to save the sums of the velocity products (i.e., $\sum u_{i} v_{i}$ ), because we can calculate them from the output turbulent shear as follows

$$
\begin{aligned}
& \left.\frac{\sum_{i=1}^{n} u_{i} v_{i}}{n}=\left\langle u^{\prime \prime} v^{\prime \prime}\right\rangle_{1}+\langle u\rangle_{1}<v\right\rangle_{1} \text {, } \\
& \sum^{2 n} u_{i} v_{i} \\
& \frac{i=n+1}{n}=\left\langle u^{\prime \prime} v^{\prime \prime}\right\rangle_{2}+\langle u\rangle_{2}\langle v\rangle_{2} \text {, etc. }
\end{aligned}
$$

Thus, the turbulent shear for the interval $t-\frac{k T}{2}$ to $t+\frac{k T}{2}$ can be postprocessed from the $k$ intermediate intervals using the following expression

$$
\begin{aligned}
& \left.\left.\left\langle u^{\prime \prime} v^{\prime \prime}\right\rangle \cong \frac{1}{k}\left\langle u^{\prime \prime} v^{\prime \prime}\right\rangle_{1}+\langle u\rangle_{1}<v\right\rangle_{1}+\left\langle u^{\prime \prime} v^{\prime \prime}\right\rangle_{2}+\langle u\rangle_{2}<v\right\rangle_{2} \\
& +\ldots+\left\langle u^{\prime \prime} v^{\prime \prime}>_{k}+\left\langle u>_{k}<v\right\rangle_{k}\right. \text { ) } \\
& \left.-\frac{1}{k^{2}}\left(\langle u\rangle_{1}+\langle u\rangle_{2}+\ldots+\langle u\rangle_{k}\right)\left(<v>_{1}+\langle v\rangle_{2}+\ldots+<v\right\rangle_{k}\right)
\end{aligned}
$$

or in summation notation,

$$
\begin{aligned}
\left\langle u^{\prime \prime} v^{\prime \prime}\right\rangle & \cong \frac{1}{k}\left(\sum_{i=1}^{k}\left\langle u^{\prime \prime} v^{\prime \prime}\right\rangle_{i}+\sum_{i=1}^{k}\langle u\rangle_{i}\langle v\rangle_{i}\right. \\
& \left.-\frac{1}{k}\left(\sum_{i=1}^{k}\langle u\rangle_{i}\right)\left(\sum_{i=1}^{k}<v{ }_{i}\right)\right) .
\end{aligned}
$$

\subsection{Turbulent Kinetic Energy}

The turbulent kinetic energy is defined as

$$
K=\frac{1}{2}\left(<u^{\prime \prime 2}>+<v^{\prime \prime 2}>+<w^{\prime \prime 2}>\right) .
$$

This quantity does not have to be calculated during calculation of the instantaneous velocity, but was done in the HYDRA code for convenience.

If a larger time interval is desired, say $k T$, the value is calculated using the above expression with the intensity values at the intermediate intervals. 


\subsection{Implementation in HYDRA Code}

The HYDRA code was modified to calculate time averages as described in the previous section. Table 1 shows a summary of the storage requirements and calculated time-averaged quantities.

TABLE 1. Summary of Storage and Output Requirements For each node and each time average interval:

\begin{tabular}{|c|c|}
\hline $\begin{array}{c}\text { Running Sum from } \\
t-\frac{T}{2} \text { to } t+\frac{T}{2}\end{array}$ & Output \\
\hline$\sum_{i} u_{i}, \sum_{i} v_{i}, \sum_{i} w_{i}$ & $\langle u\rangle,\langle v\rangle,\langle w\rangle$ \\
$\sum_{i} u_{i}^{2}, \sum_{i} v_{i}^{2}, \sum_{i} w_{i}^{2}$ & $\left.<u^{\prime 2}\right\rangle,\left\langle v^{\prime \prime 2}\right\rangle,\left\langle w^{\prime 2}\right\rangle, k$ \\
$\sum_{i} u_{i} v_{i}$, & $\left.<u^{\prime \prime} v^{\prime \prime}\right\rangle$ \\
\hline
\end{tabular}

To turn on time averaging in the HYDRA code, the control card itavg is set to 1 (default is 0 ). The time average for multiple intervals is calculated by writing the running sums to the restart dump and saving the total number of steps in the parameter itavg.

The time average velocities $\langle u\rangle,\langle v\rangle$, and $\langle w\rangle$ are written to the time history file instead of the instantaneous quantities when itavg $=1$. The plot files were modified to include two states for each time output so that all the desired time-average quantities could be output. This was required because the number of nodal variables per state is limited in a plot file due to the database limitations of the postprocessor. Table 2 is a description of the plot output and corresponding labels in the GRIZ (Speck and Dovey 1996) postprocessing routine. 
TABLE 2. 3D HYDRA Time Average Variables, and Corresponding GRIZ Labels

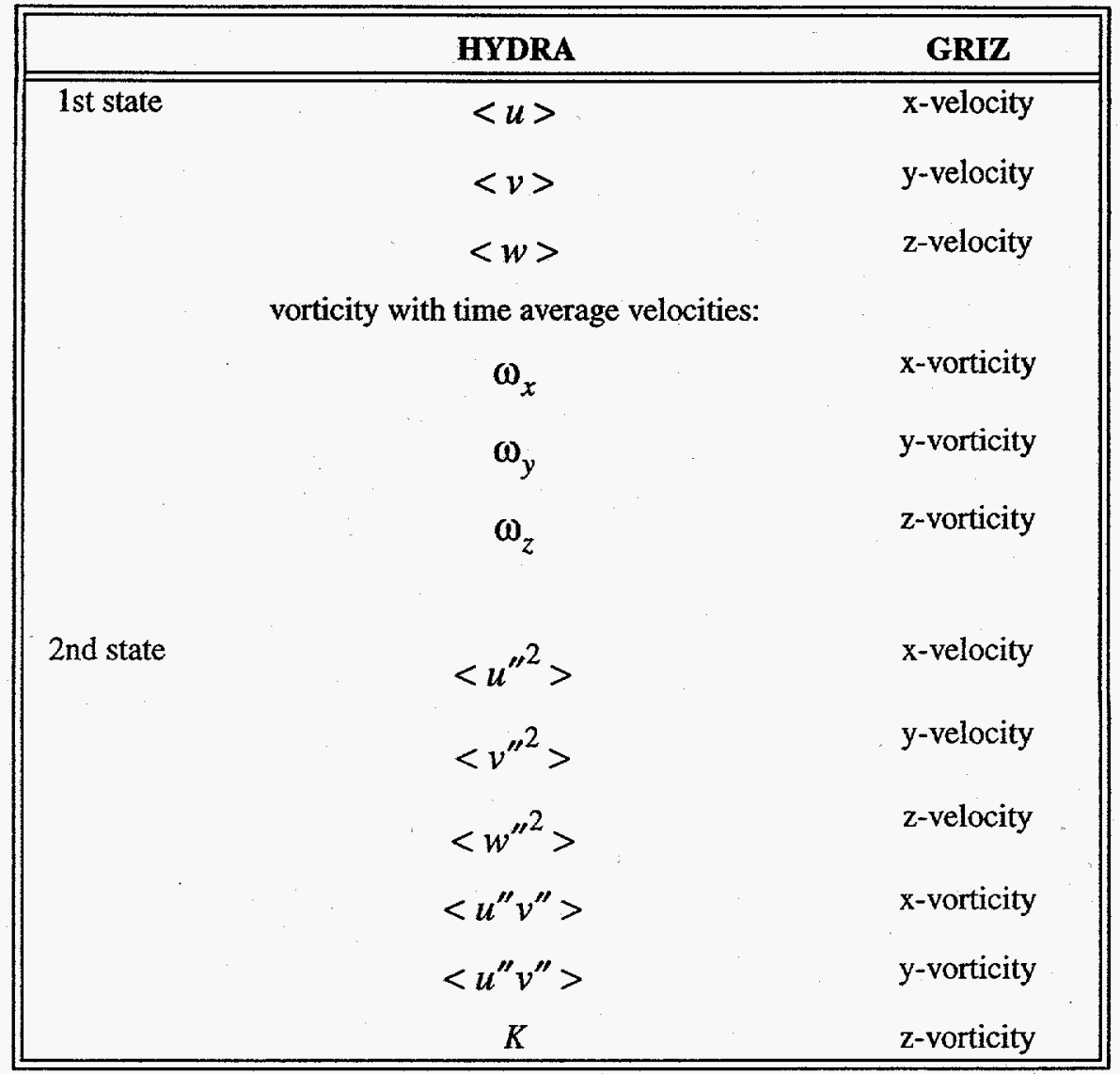

See the Appendix for a detailed description of the coding changes.

\section{References}

Hinze, J., 1975, Turbulence, McGraw-Hill.

Tennekes, H. and Lumley, J.L., 1972, A First Course in Turbulence, MIT Press.

Speck, D. E. and Dovey, D. J., 1996, "GRIZ Finite Element Analysis Results Visualization for Unstructured Grids User Manual,” LLNL, UCRL-MA-115696 Rev. 1.

Christon, M.A., 1995, "HYDRA, A Finite Element Computational Fluid Dynamics Code User Manual," LLNL, UCRL-MA-121344. 


\section{Appendix \\ Details of Coding Changes}

The following is a list of modified files with a detailed description of the code changes.

\begin{tabular}{|c|c|}
\hline STORAGE & $\begin{array}{l}\text { - pointer } 88 \text { identified for control variable itavg and summation storage tavg (nnp, ntasms) } \\
\begin{aligned} \text { itavg } & =0 \text { no time averaging } \\
& =1 \text { time average }\end{aligned} \\
\text { Note: itavg stores the stop count for restart (i.e., total steps). }\end{array}$ \\
\hline pointers.h & - tavg added to common ptrs and dimension and pointers set. \\
\hline chkpnt.F & $\begin{array}{l}\text { - subroutine ckpnt: parameter ntasms }=7 \text {, included tavg and itavg }=\text { icntl }(88) \text { in restart. } \\
\text { subroutine rstart: reads tavg if part of dump file and allocates memory, total number of steps } \\
\text { set from itavg. }\end{array}$ \\
\hline drv3d.F & $\begin{array}{l}\text { subroutine } f e 3 d \text { : keeps sums for time averages, changes for plotting, writes total number of } \\
\text { steps to itavg, changed ndim to ndof in vort } 3 d \text { calls for } u \text { and tavg, added tavg ascii output, } \\
\text { added second state plot when itavg } \neq 0,\langle u\rangle,\langle v\rangle,\langle w\rangle \text { written to time history when itavg } \\
\neq 0 \text {. }\end{array}$ \\
\hline fem3d.F & $\begin{array}{l}\text { - subroutine vort } 3 d \text { : ndof is now only dimension for } u \text { in call, changed dimension of vor and } b \\
\text { from ndof to ndim so could use routine for time averages (note: ndim wasn't used). } \\
\text { - subroutine tasums: new subroutine for calculating sums for time averages. } \\
\text { - subroutine tavgdo: new subroutine calculates time averages from sums. } \\
\text { - subroutine tavgun: undoes time average to get back sums. }\end{array}$ \\
\hline hydra.F & $\begin{array}{l}\text { - subroutine allocm: added memory allocations for time averages and since called on starting } \\
\text { run (not restart), zeroed storage. }\end{array}$ \\
\hline io.F & $\begin{array}{l}\text { - } \text { subroutine } e k o c t l: \text { added time averages. } \\
\text { - subroutine getctl: added time averages. } \\
\text { - subroutine getprm: added time averages. } \\
\text { - subroutine modprm: added time averages and expanded memory if starting run (icntl }(88)= \\
\text { 0). } \\
\text { - subroutine } t d b s t: \text { generalized for possibility of second state dump at same time (itrb }=999 \text { ). }\end{array}$ \\
\hline
\end{tabular}

